\title{
Development of Rapid Analytical Methods in the Laboratory of Pharma- ceutical Analytical Chemistry (LCAP)
}

\author{
Serge Rudaz, Laurent Geiser, Davy Guillarme, and Jean-Luc Veuthey*
}

\begin{abstract}
The use of rapid and ultra-rapid analytical methods in the pharmaceutical domain is mandatory in the drug discovery, drug development, and quality control steps. Liquid chromatography (LC) and capillary electrophoresis (CE) are therefore particularly studied in the Laboratory of Pharmaceutical Analytical Chemistry (LCAP) to perform fast and ultra-fast analyses of drugs present in different matrices. Due to its sensitivity and selectivity, mass spectrometry (MS) is considered today as the gold standard in pharmaceutical analysis and has been generally used as the detector of choice for performing rapid analyses. This article gives a survey of the applied strategies achieved in the LCAP for the last five years such as: use of new chromatographic supports and small columns in LC; development of column switching techniques with extraction supports compatible with the direct injection of biological matrices; use of immobilized enzyme reactors for rapid protein digestion; enhancement of electrophoretic mobility and development of different injection procedures in CE.
\end{abstract}

Keywords: Capillary electrophoresis · Fast analysis · Liquid chromatography · Mass spectrometry · Sample preparation - Ultra-fast analysis

\section{Introduction}

The commercialization of a drug product is a long and tedious task which can take about 10-15 years from the development of a lead compound to its commercialization. Therefore, one of the main objectives of the pharmaceutical industry is to reduce this time period. For this purpose, highthroughput discovery and screening methods have been developed and as a result the analytical laboratory is faced with a great

${ }^{*}$ Correspondence: Prof. J.-L. Veuthey

School of Pharmaceutical Sciences

Ecole de Pharmacie Genève-Lausanne

Laboratoire de Chimie Analytique Pharmaceutique

University of Geneva

Quai Ernest Ansermet 30

$\mathrm{CH}-1211$ Geneva 4

Tel.: + 41223796336

Fax: + 41223796808

E-Mail: Jean-Luc.Veuthey@pharm.unige.ch number of samples. Furthermore, the delay to provide analytical responses should be as short as possible. Therefore, new rapid and efficient procedures have to be used for qualitative and quantitative analysis.

The analytical techniques mainly used to screen and perform quantitative determination are immunoassay, spectroscopy, and separation tools such as chromatography and electrophoresis. The Laboratory of Pharmaceutical Analytical Chemistry (LCAP) is mainly involved in the development of liquid chromatography (LC) and electrophoretic techniques such as capillary electrophoresis (CE) for the analysis of drugs and metabolites in pharmaceutical formulations as well as in biological matrices. With both techniques coupled with different methods of detection, it is possible to perform very rapid, efficient, and sensitive analyses.

\section{Rapid Liquid Chromatography}

LC is often considered as the method of choice for quantitative determination of drugs and related substances. However, conventional analysis times are usually longer than $10 \mathrm{~min}$. As reported in the literature, rapid or fast methods are relative terms depending on the analyst and the requirement. Ultra-fast or ultra-rapid methods can be defined by cycle times less than 1 min (including column reconditioning), and fast or rapid methods by cycle times less than 5 min. In order to carry out rapid analyses, different strategies can be applied as presented in Fig. 1.

The use of short columns $(3-5 \mathrm{~cm})$ to decrease the analyte retention volume and the increase of the flow rate are the simplest approaches to reduce the analysis time. However, both can compromise the chromatographic performance. Because the column reduction could lead to an important loss in efficiency, particle size should be simultaneously decreased or other chromatographic material could be used.

Thus, for the former approach new supports have been developed such as small $(3.5 \mu \mathrm{m}$ and sub-2 $\mu \mathrm{m})$ silica-based particles. According to the Van Deemter curve, efficiency and optimal velocity are increased with very small particles (sub-2 $\mu \mathrm{m}$ ), allowing a separation with good resolution in a short analysis time. Furthermore, due to a faster mass transfer, velocity can be increased beyond its optimal value whilst maintaining a good efficiency (Fig. 2). However, these conditions can generate a high back pressure ( $>400$ bar) not compat- 


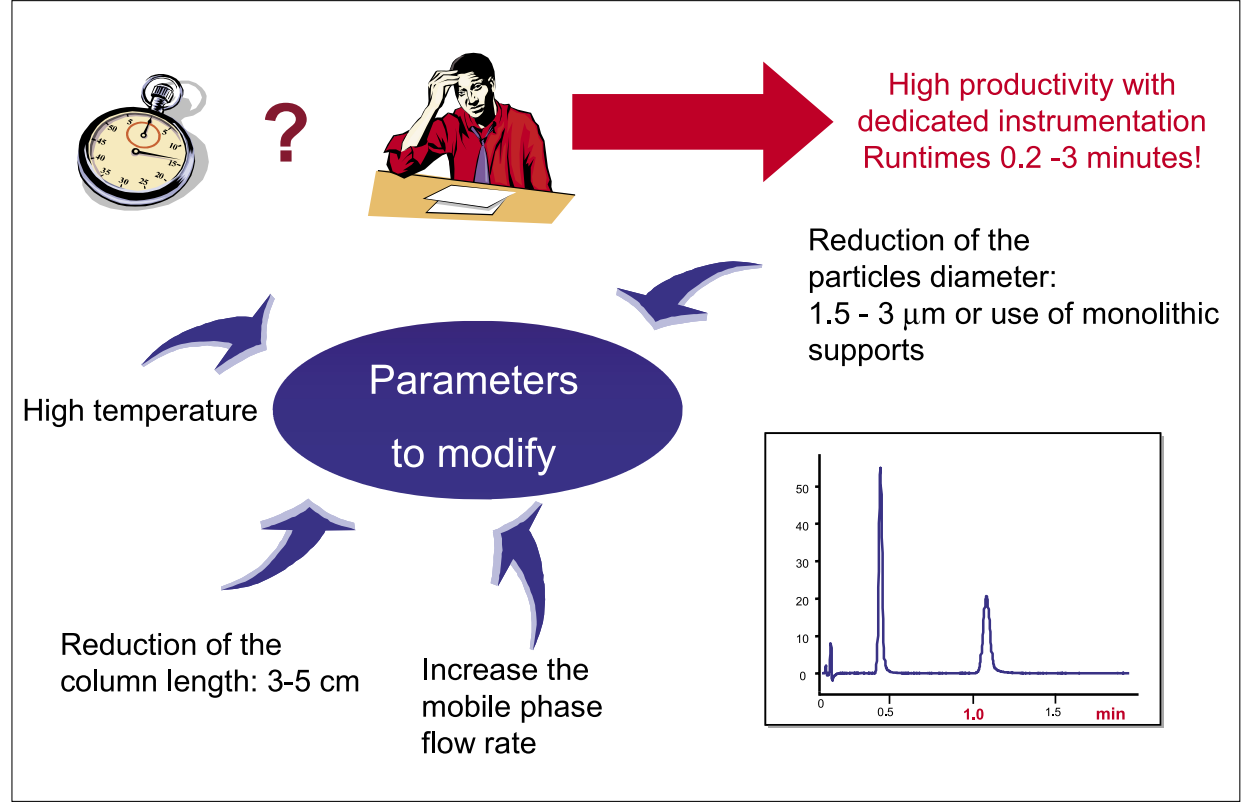

Fig. 1. Strategies for performing rapid liquid chromatography

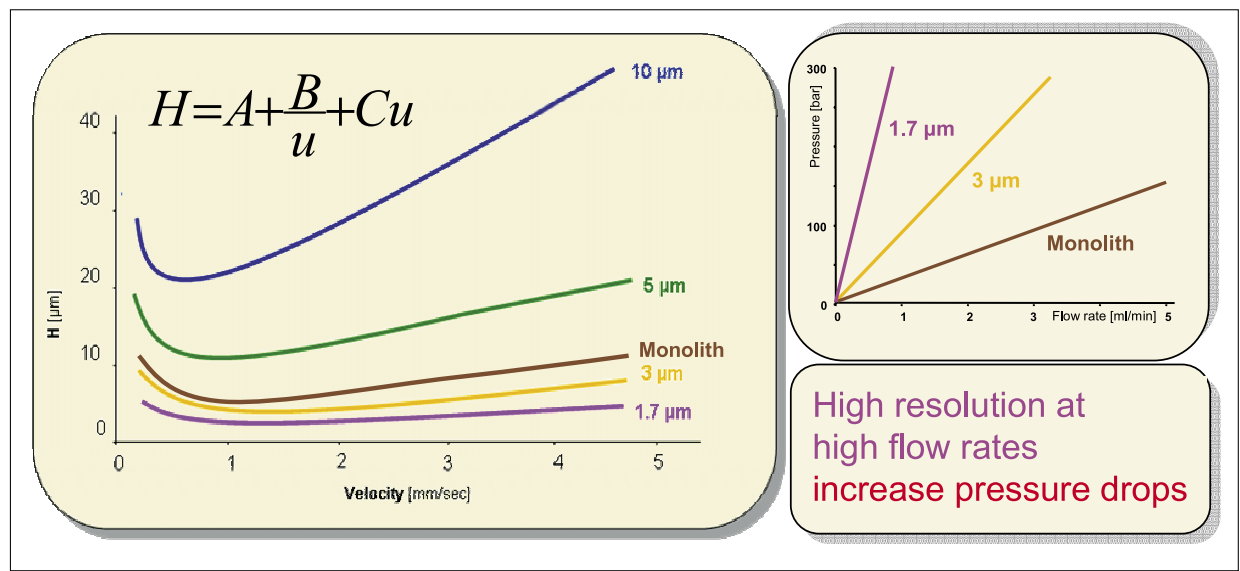

Fig. 2. Van Deemter curves and generated back pressures as a function of particle size. Adapted from [1].

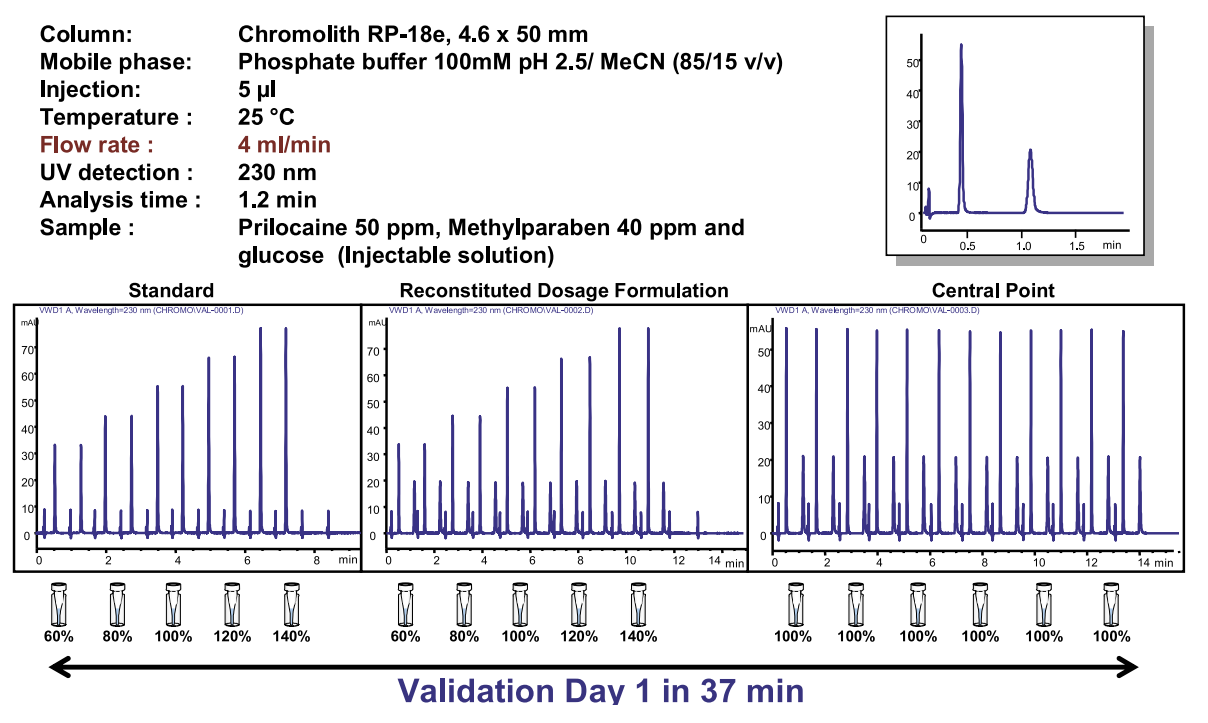

Fig. 3. Rapid validation of prilocaine in pharmaceutical formulations by LC-UV with monolithic column ible with conventional instrumentation. To overcome this problem, an inhomogeneous particle size distribution with sub- $2 \mu \mathrm{m}$ and higher diameters can be used. It is also possible to perform analyses with high back pressure compatible instrumentation (i.e. 1000 bar) as the UPLC (ultra high pressure liquid chromatography) system recently commercialized.

Another approach concerns the use of monolith instead of particles packed support. Monolithic rods, made of silica or polymeric material, can accept high flow rates (typically 3 to 10 times larger than conventional flow rates) without generating high back pressure and with efficiency and resolution comparable to silica particles of ca. $3 \mu \mathrm{m}$ (Fig. 2). This particular behavior is due to its bi-modal structure with macropores and mesopores.

The LCAP has already developed different methods for the rapid analysis of drugs with the above-mentioned strategies [2][3]. Research is in progress to determine advantages and drawbacks in terms of efficiency, robustness, cost, and sensitivity. As an example, a method on commercially available monolithic support for the rapid analysis of prilocaine in pharmaceutical formulation is presented in Fig. 3. The short analysis time allows a great number of analyses to be performed per unit of time and the very fast validation of the method.

Finally, high column temperature $(>100$ ${ }^{\circ} \mathrm{C}$ ) can be used to perform rapid analysis since viscosity and therefore back pressure decrease. Furthermore, as already reported in the literature, efficiency as well as mass transfer is enhanced with temperature, permitting the application of higher mobile phase velocity. Some drawbacks have to be mentioned in terms of stationary phase choice, compound stability, and dedicated instrumentation. This strategy is also implemented at the LCAP and rapid methods at high column temperature are under development. All these approaches could be summarized in the following table (Table). There is always a compromise between advantages and drawbacks, such as column back pressure, analysis time and column efficiency.

\section{Rapid Sample Preparation}

With new rapid and ultra-rapid analytical tools, the sample preparation step needs to be reconsidered since it remains often the rate limiting step of the analytical process. Especially with LC coupled with mass spectrometry (MS), methods with high sensitivity and selectivity can be obtained with cycle times lower than $5 \mathrm{~min}$. Therefore, the LCAP has developed different generic methods entirely automated including the sample preparation coupled to LC-MS 
Table. Advantages and drawbacks of different approaches used in LC to reduce the analysis time

$\begin{array}{lll}\text { Approach } & \text { Advantages } & \text { Drawbacks } \\ \text { Short column } & \begin{array}{l}\text { Conventional equipment } \\ \text { Many stationary phases available }\end{array} & \begin{array}{l}\text { Low efficiency and resolution } \\ \text { Extra column band broadening }\end{array} \\ \text { Monolithic columns } & \begin{array}{l}\text { Conventional equipment } \\ \text { Low back pressure }\end{array} & \begin{array}{l}\text { Few columns commercially } \\ \text { available }\end{array} \\ \text { Ultra-high pressure LC } & \text { High efficiency with small particles } & \begin{array}{l}\text { Specialized equipment } \\ \text { Few stationary phases available }\end{array} \\ \text { High-temperature LC } & \begin{array}{l}\text { Low back pressure } \\ \text { High efficiency }\end{array} & \begin{array}{l}\text { Specialized equipment } \\ \text { Specialized stationary phases }\end{array}\end{array}$

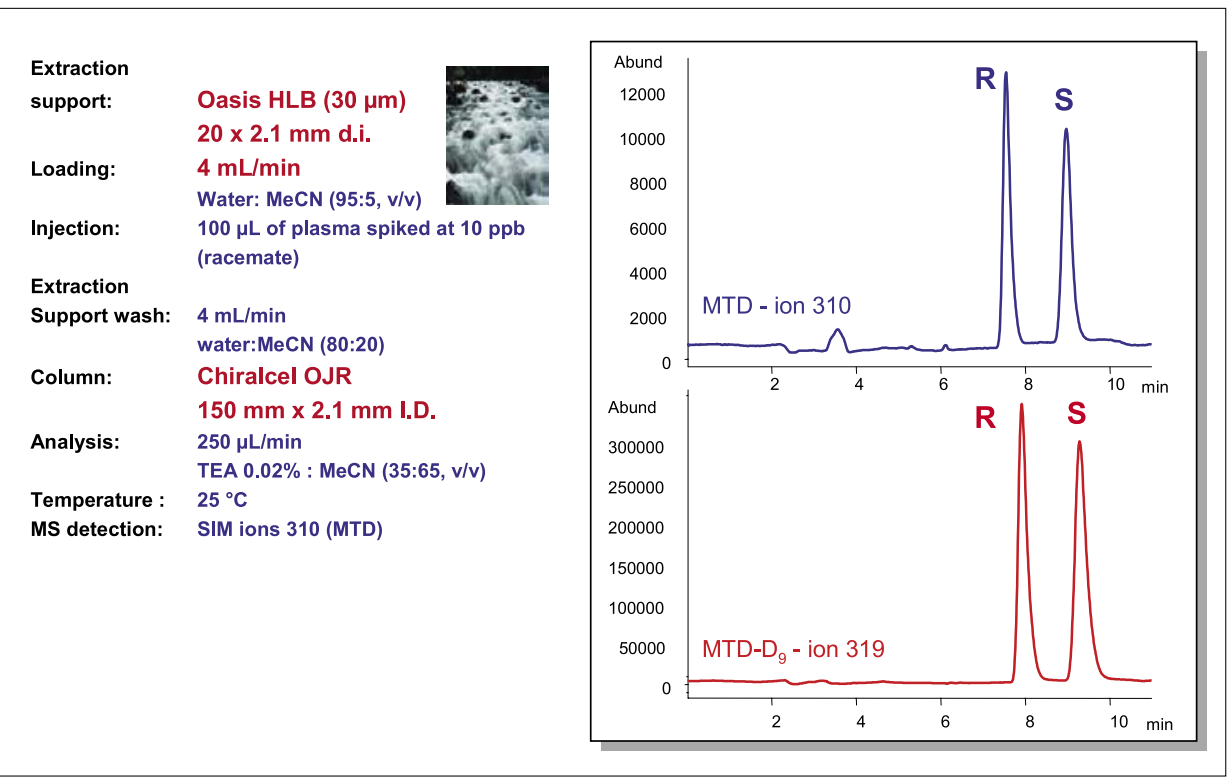

Fig. 4. Automated stereoselective analysis of methadone enantiomers

analysis for the analysis of biological matrices [4-7]. For this purpose, the columnswitching approach was used with packing materials compatible with the direct injection of biological matrices.

Restricted access material (RAM), large particles support (LPS) and monolithic support were tested as extraction supports for the rapid elimination of proteins and other endogenous material and the retention of different drugs and metabolites with the column-switching approach [8-10]. These supports were compared in terms of sample preparation time, protein elimination efficiency as well as ion suppression effect when coupled with an analytical column and MS detection [11][12]. This strategy has been applied with success to a great number of drugs and metabolites and it was possible to inject in these supports numerous volumes of plasma and serum samples without losing performance. Due to the versatility of the column-switching approach, a procedure was also validated for the sepa- ration of methadone enantiomers in plasma samples [13]. A polymeric LPS extraction column, compatible with an alkaline mobile phase, coupled to a chiral stationary phase (Chiralcel OJR) allowed the rapid stereoselective analysis of methadone enantiomers in plasma samples as shown in Fig. 4.

Beside sample preparation, the column-switching approach was also used at the LCAP for the rapid on-line digestion of proteins. Indeed, the growing interest in proteomics induces the development of robust, automated, sensitive and highthroughput analytical tools. Rapid protein identification via peptide mapping is employed for a large range of applications such as detection of pathological changes in proteins, detection of post-translational modification and quality control of recombinant proteins. In these cases, peptides fragments are separated and sequenced by liquid chromatography coupled with electrospray ionization tandem mass spectrometry (LC-ESI-MS/MS). Trypsin is a widely used proteolytic enzyme that can be used in solution or in solid phase. In this case, trypsin is immobilized on a solid support (IMER $=$ immobilized enzyme reactor) allowing a rapid digestion (minutes instead of hours) due to an elevated enzyme/protein ratio. Furthermore, it can be re-used several times and autoprotolysis is reduced or avoided. Thus, IMER is cost-effective and compatible with high-throughput procedures. In collaboration with the University of Pavia (Italy), a bio-reactor with trypsin immobilized on a silica monolithic support for the rapid on-line protein digestion and peptide analysis by LC-MS-MS was developed [14]. The instrumental set-up is reported in Fig. 5. The influence of various parameters on enzymatic activity was investigated following a multivariate approach (experimental design). After optimization, tryptic digestions of myoglobin performed on-line and off-line were compared. The sequence coverage obtained by both procedures was identical while the total analysis time was largely reduced.

\section{Rapid Capillary Electrophoresis}

Capillary electrophoresis is now recognized as a complementary analytical technique to liquid chromatography. Both methods are considered as orthogonal since separation in LC is based on adsorption and/or partition mechanisms while electrophoresis selectivity is obtained by the differential migration of charged species under an electric field. Therefore, the separation patterns obtained by CE and LC are orthogonal, making the combined use of these two techniques a powerful analytical tool. In the pharmaceutical domain, $\mathrm{CE}$ has rapidly become successful due to its very high efficiency, short analysis time and method development, simple instrumentation, low sample and solvent consumption as well as reduced operating costs. Another important aspect for emphasizing the implementation of capillary electrophoresis is its ability to separate numerous compounds of pharmaceutical interest. As shown in Fig. 6, several approaches have been reported to achieve fast separation on commercially available instruments, such as increasing the electric field through a reduction of the total capillary length at high applied voltage and decreasing the effective length by using the short-end injection technique. It is also possible to reduce the analysis time by increasing the electroosmotic mobility through a dynamical coating procedure of the capillary or by performing multiple injections. Hence, with conventional instrumentation, it is also possible to carry out analyses with runtimes of less than $1 \mathrm{~min}$ at very low cost. 


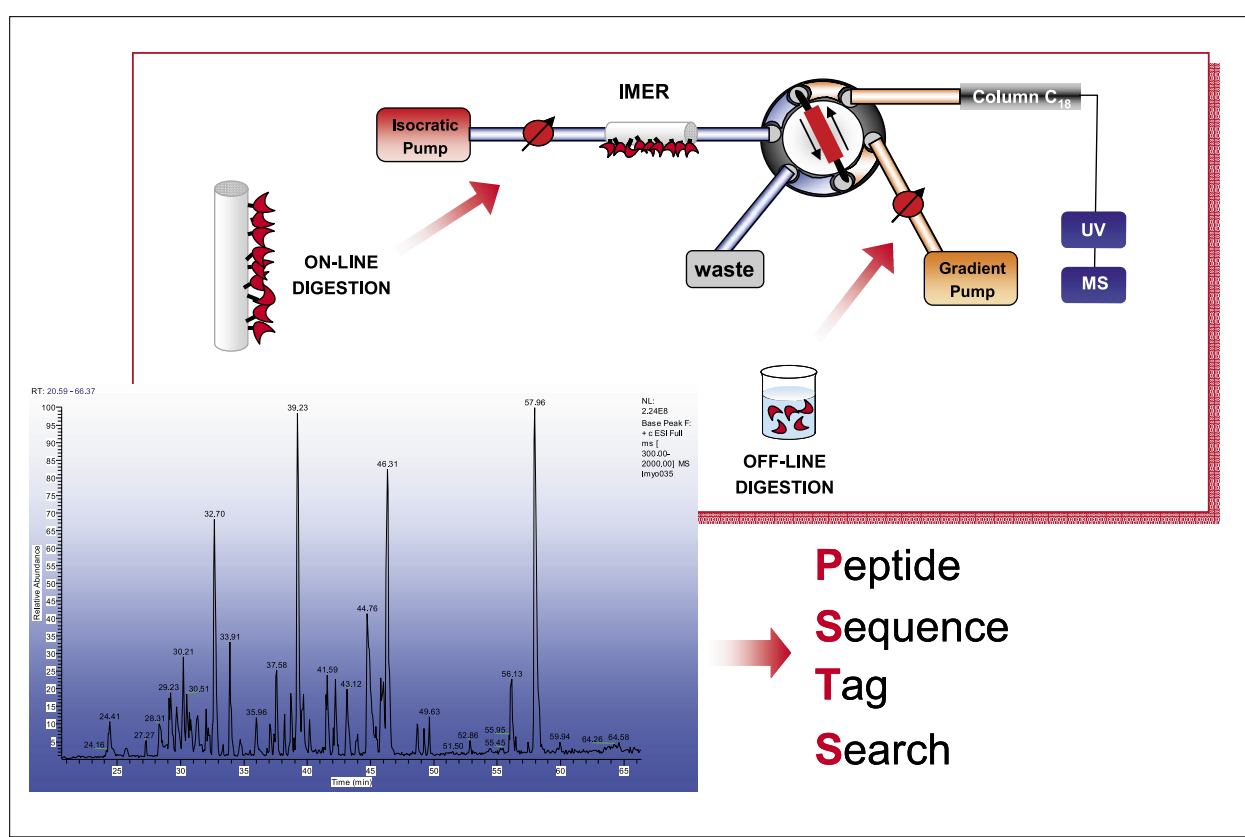

Fig. 5. Set-up for on-line trypic digestion of myoglobin and PSTS: IMER trypsin bioreactor $(25 \times$ $4.6 \mathrm{~mm} \mathrm{I.D)}, \mathrm{C}_{18}$ trapping column $(10 \times 4.6 \mathrm{~mm} \mathrm{I.D)}), \mathrm{C}_{18}$ analytical column $(100 \times 2.1 \mathrm{~mm} \mathrm{I.D)}) \mathrm{MS}$ detection: LCQ DECA ion trap

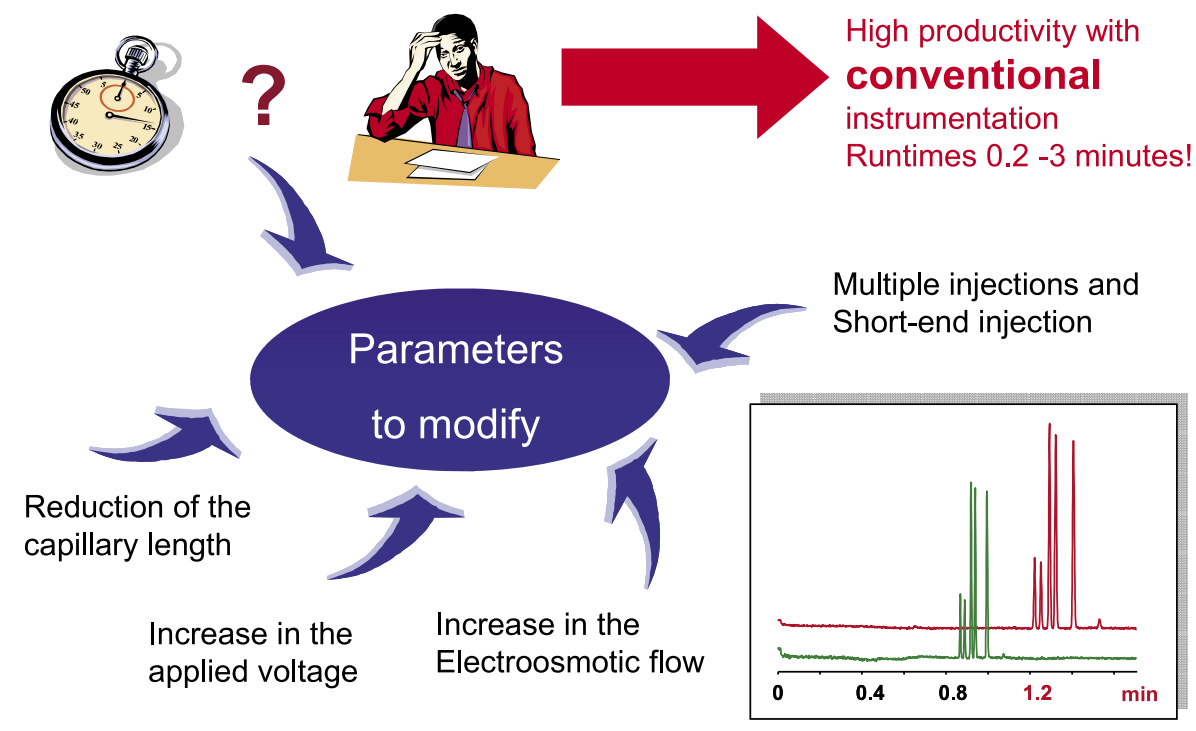

Fig. 6. Strategies for performing rapid capillary electrophoresis

Recently, a systematic investigation to compare quantitative performances of the aforementioned approaches with a conventional CE procedure for the analysis of a pharmaceutical formulation was performed [15][16]. The migration time and the total analysis time (TAT) were also studied and it was clearly demonstrated that excellent validation results were obtained with all tested approaches. From the results, it appeared that the application of a high electric field was suitable for reducing the TAT in comparison with a conventional method (ca. 80\%). On the other hand, lower precision performances due to Joule heating were observed. Therefore, this approach is better adapted to low conductivity buffer electrolyte solutions as used in nonaqueous capillary electrophoresis (NACE) such as reported in Fig. 7 for the ultra-fast separation of eight tropane alkaloids [17].

The short-end injection technique gave also satisfactory results in terms of precision, accuracy, and analysis time. The electroosmotic flow (EOF) increase by the dynamic coating procedure gave relevant results in efficiency and validation data. Furthermore, this procedure permits migration times to be stabilized by both preventing interactions between compounds and the capillary wall and exhibiting a constant elevated EOF. The coating procedure can be performed prior to a sequence and more than 30 analyses can be carried out without loss of analytical performance. In our opinion, this procedure is considered as the method of choice for conducting rapid routine analyses. Finally, the multiple injections procedure was tested and the TAT per sample, which includes the analysis time, the rinsing procedure, the injection and the permutation vial, was largely decreased. The validation and separation performances were quite similar to those obtained with conventional and dynamic coating procedures.

Chiral separation is certainly the domain where the high efficiency obtained in $\mathrm{CE}$ presents major advantages versus chromatography. Effectively, a large number of selectors are commercially available and the time of method development is really short in comparison with liquid chromatography. Challenging separations such as chiral separation can be performed very rapidly with a high electric field on short capillary as reported in Fig. 8.

Finally, CE can also be coupled with mass spectrometry to perform rapid analyses. CE-MS combines the advantages of both techniques. The coupling is commercially available and is generally constituted of modified electrospray source with a coaxial sheath liquid interface. Different parameters have to be adjusted such as the nebulizing gas pressure, the composition and the flow rate of the sheath liquid and the position of the capillary in the interface as well as MS parameters [19][20]. CE-MS like CE-UV is well adapted to the analysis of charged species as well as of neutral compounds. For the latter, capillary electrochromatography (CEC) is generally used instead of micellar electrokinetic chromatography (MEKC). It is also necessary to replace conventional with volatile buffers. In the LCAP, several methods with a simple quadrupole were developed but other analyzers can be used such as a simple or triple quadrupole, ion trap or time of flight (TOF).

Different CE-MS methods were validated with aqueous and non aqueous solvents for the analysis of drugs and metabolites in different matrices such as pharmaceutical formulations and biological fluids after a simple liquid-liquid or solid-phase extraction [21-24]. Sensitivity was in all cases improved in comparison with CE-UV. Furthermore, time of analysis can be reduced, since MS affords a supplementary selectivity. Indeed MS is not only a detection but also a separation technique resulting in $2 \mathrm{D}$ separation when coupled with CE.

\section{Perspectives}

The development of chromatographic supports and instrumentation compatible 


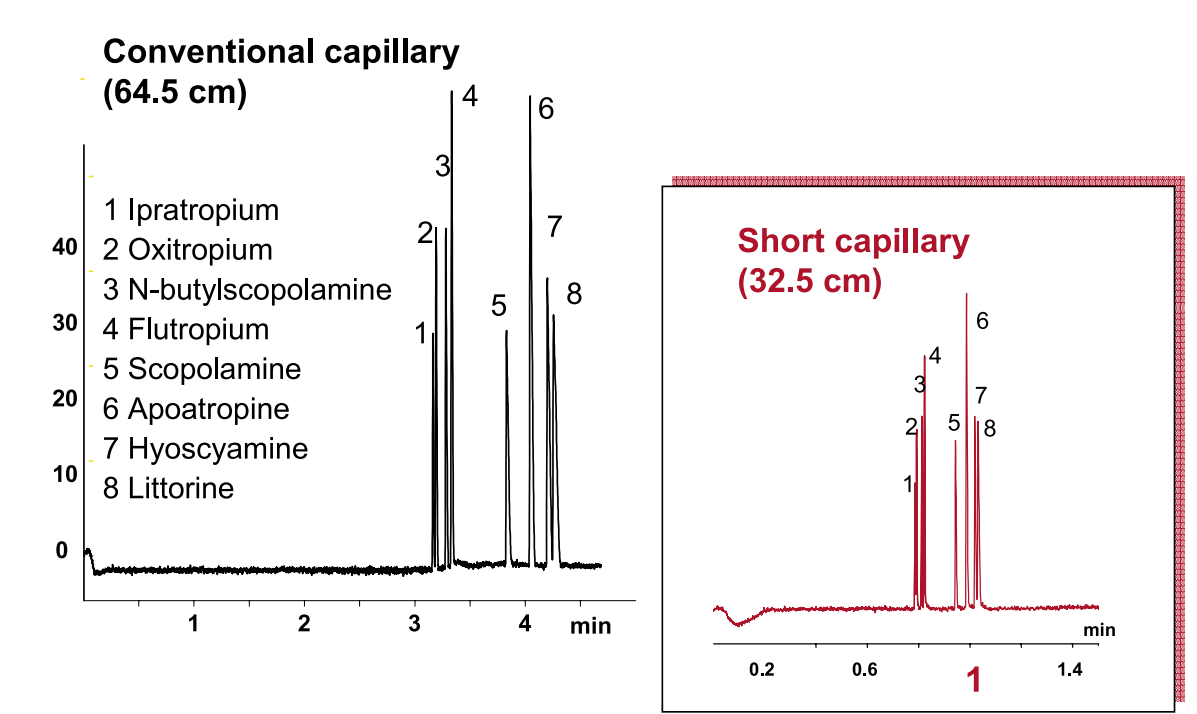

Fig. 7. Rapid non-aqueous $\mathrm{CE}$ of tropane alkaloids [17]. Experimental conditions: $25: 75$ methanolacetonitrile with $25 \mathrm{mM}$ ammonium acetate and $1 \mathrm{M}$ acetic acid, $\mathrm{T}=25^{\circ} \mathrm{C}, \mathrm{U}=30 \mathrm{kV}$.
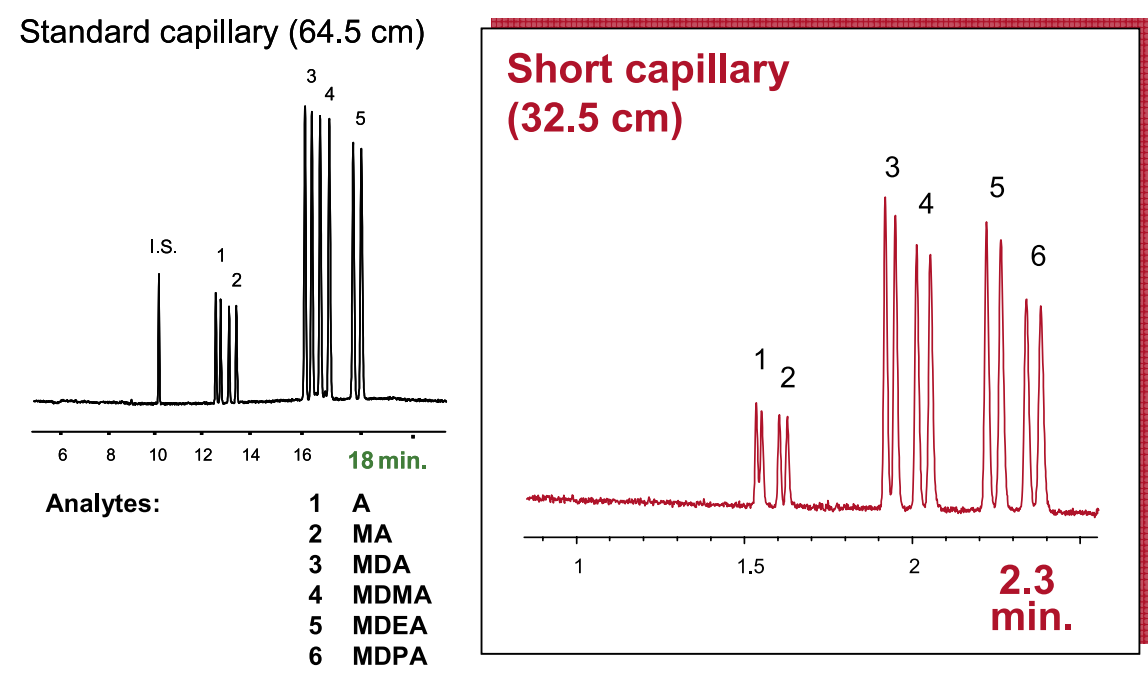

Fig. 8. Rapid chiral CE of amphetamines [18]. Experimental conditions: $118 \mathrm{mM}$ tris-phosphate buffer at $\mathrm{pH} 3.5$, with hydroxypropyl- $\beta$ cyclodextrin $16 \mathrm{mM}, \mathrm{T}=28^{\circ} \mathrm{C}, \mathrm{U}=25 \mathrm{kV}$.

with rapid and ultra-rapid LC separations will certainly grow in the future. In this context, the LCAP is investigating the different proposed approaches to compare them in terms of cost, robustness, sensitivity, and compatibility with different detection systems. In CE, some new tools such as the multiplexing capillary array instrument can also be of great interest to perform a large number of analyses per time unit. This strategy, already commercialized, can be used to determine physicochemical properties of drug products ( $\mathrm{pKa}$ and $\log \mathrm{P}$ values) as well as to screen rapidly chiral selectors.

New and future developments of LC and $\mathrm{CE}$ will certainly be dedicated to minia- turization. The nano-technologies can offer several advantages in terms of cost reduction and space saving. They can also be used onsite as a complete analytical tool integrating the sample preparation, separation and detection. This concept was developed by Widmer and co-workers [25] a decade ago and called micro total analysis system $(\mu$ TAS). Recently, different procedures with nano-technologies were published in the literature especially dedicated to genomics and proteomics. But new developments of nano-CE and nano-LC separations have appeared recently also in pharmaceutical analysis. Therefore, the LCAP, in collaboration with DiagnoSwiss (Monthey, Swit- zerland) is working on a $\mathrm{KTI} / \mathrm{CTI}$ project (OFFT, Switzerland) about the coupling of chip-CE and chip-LC with MS and other detection modes.

Received: April 15, 2005

[1] D. Lubda, K. Cabrera, W. Kraas, C. Schaefer, D. Cunningham, $L C G C N$. $A M$. 2001, 19, 1186.

[2] B. Kaufmann, S. Souverain, S. Cherkaoui, P. Christen, J.L. Veuthey, Chromatographia 2002, 56, 137.

[3] D. Guillarme, S. Heinisch, J.L. Rocca, J. Chromatogr. A 2004, 1052, 39.

[4] D. Ortelli, S. Rudaz, S. Souverain, J.L. Veuthey, J. Sep. Sci. 2002, 25, 222.

[5] S. Souverain, S. Rudaz, D. Ortelli, E. Varesio, J.L. Veuthey, J. Chromatogr. B 2003, $784,117$.

[6] S. Souverain, S. Rudaz, J.L. Veuthey, G.I.T. Lab. J. Eur. 2003, 7, 80.

[7] S. Souverain, S. Rudaz, J.L. Veuthey, Chromatographia 2003, 57, 569.

[8] S. Souverain, S. Rudaz, J.L. Veuthey, J. Chromatogr. B 2004, 801, 141.

[9] J.L. Veuthey, S. Souverain, S. Rudaz, Ther Drug Monit. 2004, 26, 161.

[10] S. Souverain, M. Mottaz, S. Cherkaoui, J.L. Veuthey, Anal. Bioanal. Chem. 2003, $377,880$.

[11] S. Souverain, S. Rudaz, J.L. Veuthey, J. Chromatogr. A 2004, 1058, 61.

[12] S. Souverain, S. Rudaz, J.L. Veuthey, J. Pharm. Biomed. Anal. 2004, 35, 913.

[13] S. Souverain, C. Eap, J.L. Veuthey, S. Rudaz, Clin. Chem. Lab. Med. 2004, 41, 1615.

[14] E. Calleri, C. Temporini, E. Perani, C. Stella, S. Rudaz, D. Lubda, G. Mellerio, J. L. Veuthey, G. Caccialanza, G. Massolini, J. Chromatogr. A 2004, 1045, 99.

[15] L. Geiser, S. Rudaz, J.L. Veuthey, Electrophoresis 2003, 24, 3049.

[16] L. Geiser, S. Rudaz, J.L. Veuthey, Electrophoresis, in press.

[17] S. Cherkaoui, L. Geiser, J.L. Veuthey, Chromatographia 2000, 52, 403.

[18] E. Varesio, J.Y. Gauvrit, R. Longeray, P. Lanteri, J.L. Veuthey, Electrophoresis 1997, 18, 931.

[19] S. Rudaz, S. Cherkaoui, J.Y. Gauvrit, P. Lanteri, J.L. Veuthey, Electrophoresis 2001, 22, 3316.

[20] E. Varesio, S. Cherkaoui, J.L. Veuthey, J. High. Res. Chrom. 1998, 21, 653.

[21] S. Cherkaoui, S. Rudaz, E. Varesio, J.L. Veuthey, Chimia 1999, 53, 501.

[22] S. Cherkaoui, S. Rudaz, J.L. Veuthey, Electrophoresis 2001, 22, 491.

[23] S. Cherkaoui, S. Rudaz, E. Varesio, J.L. Veuthev Electrophoresis 2001, 22, 3308.

[24] L. Geiser, S. Cherkaoui, J.L. Veuthey, J. Chromatogr. A 2002, 979, 389.

[25] A. Manz, N. Graber, H.M. Widmer, Sens. Actuator B-Chem. 1990, B1, 244. 